Pacific Journal of Mathematics

ON SOME TOTALLY ERGODIC FUNCTIONS 


\title{
ON SOME TOTALLY ERGODIC FUNCTIONS
}

\author{
WOJCIECH CHOJNACKI \\ Dedicated to Dagmara Klim and Nina Tomaszewska
}

\begin{abstract}
We study some classes of totally ergodic functions on locally compact Abelian groups. Among other things, we establish the following result: If $R$ is a locally compact commutative ring, $\mathscr{R}$ is the additive group of $R, \chi$ is a continuous character of $\mathscr{R}$, and $p$ is the function from $\mathscr{R}^{n}(n \in \mathbb{N})$ into $\mathscr{R}$ induced by a polynomial of $n$ variables with coefficients in $R$, then the function $\chi \circ p$ either is a trigonometric polynomial on $\mathscr{R}^{n}$ or all of its Fourier-Bohr coefficients with respect to any Banach mean on $L^{\infty}\left(\mathscr{R}^{n}\right)$ vanish.
\end{abstract}

1. Introduction. Let $G$ be a locally compact Abelian group, $\lambda_{G}$ be the Haar measure in $G$, and $L^{\infty}(G)$ be the space of all classes of complex-valued $\lambda_{G}$-measurable $\lambda_{G}$-essentially bounded functions on $G$ endowed with the $\lambda_{G}$-essential supremum norm.

A linear continuous functional $m$ on $L^{\infty}(G)$ is called a Banach mean on $L^{\infty}(G)$ if it satisfies the following conditions:

(i) $m(1)=1=\|m\|$,

(ii) $m\left(T_{a} f\right)=m(f)$ for each $a \in G$ and each $f \in L^{\infty}(G)$, where $T_{a} f(b)=f(a+b)$ for any $b \in G$.

When $G$ is finite, there is precisely one Banach mean on $L^{\infty}(G)$. When $G$ is infinite, then the set of all Banach means on $L^{\infty}(G)$ has at least the cardinality of the continuum (cf. [6, Propositions 22.26 and 22.41]).

Let $\widehat{G}$ be the dual group of $G$. Given $f \in L^{\infty}(G), \chi \in \widehat{G}$, and a Banach mean $m$ on $L^{\infty}(G)$, let $\mathscr{F}_{m} f(\chi)$ stand for the Fourier-Bohr coefficient of $f$ at $\chi$ with respect to $m$, defined to be $m(f \bar{\chi})$.

A function $f$ in $L^{\infty}(G)$ is said to be ergodic if its mean value $m(f)$ is independent of the choice of the Banach mean $m$ on $L^{\infty}(G)$. A function $f$ in $L^{\infty}(G)$ is said to be totally ergodic if, for every $\chi \in \widehat{G}$, the function $f \chi$ is ergodic (cf. $[7,8]$ ). Let $E(G)$ be the space of all ergodic functions in $L^{\infty}(G), T E(G)$ be the space of all totally ergodic functions in $L^{\infty}(G)$, and $T E_{0}(G)$ be the subspace of $T E(G)$ consisting of those $f \in L^{\infty}(G)$ for which $\mathscr{F}_{m} f(\chi)=0$ for any $\chi \in \widehat{G}$ and any Banach mean $m$ on $L^{\infty}(G)$. Let $P(G)$ be the space of all 
functions in $L^{\infty}(G)$ which, to within modification on a $\lambda_{G}$-null set, are trigonometric polynomials on $G$. It is readily verified that

$$
P(G) \subset T E(G)
$$

and that

$$
P(G) \cap T E_{0}(G)=\{0\} .
$$

The chief aim of the present paper is to show that certain subsets of $L^{\infty}(G)$, determined by conditions formulated with use of some coboundary operator, are contained in $P(G) \cup T E_{0}(G)$. One consequence of the main result about those subsets reads as follows: If $R$ is a locally compact commutative ring, $\mathscr{R}$ is the additive group of $R, \chi$ is an element of $\widehat{\mathscr{R}}$, and $p$ is the function from $\mathscr{R}^{n}(n \in \mathbb{N})$ into $\mathscr{R}$ induced by a polynomial of $n$ variables with coefficients in $R$, then the function $\chi \circ p$ is an element either of $P\left(\mathscr{R}^{n}\right)$ or of $T E_{0}\left(\mathscr{R}^{n}\right)$.

2. Preliminaries. Given a set $A, \# A$ denotes the cardinality of $A$. If $A$ is subset of a larger set, then $1_{A}$ stands for the characteristic function of $A$.

Given $a \in G$ and a subset $A$ of $G$, let

$$
a+A=\{b \in G: b-a \in A\} \text {. }
$$

A complex-valued function $f$ on $G$ with values of unit modulus will be called unitary. A function in $L^{\infty}(G)$ which, to within modification on a $\lambda_{G}$-null set, is unitary will be called almost unitary. We denote by $U(G)$ the set of all almost unitary functions in $L^{\infty}(G)$, and write $U_{0}(G)$ for $U(G) \cap P(G)$.

Let $f$ be function in $U(G)$. For each $a \in G$, put

$$
\delta_{a} f=\bar{f} \cdot T_{a} f
$$

and, for any $a_{1}, \ldots, a_{n} \in G$, set inductively

$$
\delta_{a_{1} \cdots a_{n}} f=\delta_{a_{n}}\left(\delta_{a_{1} \cdots a_{n-1}} f\right) .
$$

For each $1 \leq p<+\infty$, let $L^{p}(G)$ be the $p$ th Lebesgue space based on $\lambda_{G}$.

Given $f \in L^{1}(G)$, let $\mathscr{F} f$ denote the Fourier transform of $f$, defined by

$$
\mathscr{F} f(\chi)=\int_{G} f(a)(a,-\chi) d \lambda_{G}(a) \quad(\chi \in \widehat{G}) ;
$$

here $(a,-\chi)$ stands for the value of the character $-\chi$ at $a$. Let $\sigma(f)$ denote the spectrum of $f$, that is, the support of $\mathscr{F} f$. 
If $f \in P(G)$ is $\lambda_{G}$-essentially equal to a trigonometric polynomial $\sum_{\chi \in \hat{G}} a_{\chi} \chi$, then the set $\left\{\chi \in \widehat{G}: a_{\chi} \neq 0\right\}$ will also be denoted as $\sigma(f)$ and referred to as the spectrum of $f$.

For each $n \in \mathbb{N}$, let

$$
P_{n}(G)=\{f \in P(G): \# \sigma(f) \leq n\}
$$

and

$$
U_{n}(G)=\left\{f \in U(G): \delta_{a_{1} \cdots a_{n}} f \in P(G) \text { for } a_{1}, \ldots, a_{n} \in G\right\} .
$$

For each $m \in \mathbb{N}$, let

$$
U_{0, m}(G)=U(G) \cap P_{m}(G)
$$

and, for any $n, m \in \mathbb{N}$, let

$$
U_{n, m}(G)=\left\{f \in U(G): \delta_{a_{1} \cdots a_{n}} f \in P_{m}(G) \text { for } a_{1}, \ldots, a_{n} \in G\right\} .
$$

Given a probability triple $(\Omega, \mathscr{B}, \mathbb{P})$ and a $\sigma$-subalgebra $\mathscr{A}$ of $\mathscr{B}$, we write $\mathbb{E}^{\mathscr{A}}$ for the conditional expectation operator relative to $\mathscr{A}$.

For a subset $A$ of a vector space, the linear span of $A$ is denoted by span $A$.

For a subset $A$ of a set $B$ with a topology, we denote by $\bar{A}$ the closure of $A$ in $B$.

3. A characterization of $U_{0}(G)$. In this section, we give a characterization of the set $U_{0}(G)$ for an arbitrary locally compact Abelian group $G$. We start with the following.

Proposition 3.1. Let $G$ be a locally compact Abelian group such that $\widehat{G}$ is torsion-free. Then

$$
U_{0}(G)=U_{0,1}(G)
$$

Proof. Clearly, it suffices to show that $U_{0}(G) \subset U_{0,1}(G)$.

Let $f$ be a function in $U_{0}(G)$ and let $\sum_{i=1}^{n} a_{i} \chi_{i}$ be the trigonometric polynomial on $G \lambda_{G}$-essentially equal to $f$, with $\sigma(f)=\left\{\chi_{i}: 1 \leq\right.$ $i \leq n\}$. Suppose that $n \geq 2$. Let $\Gamma$ be the subgroup of $\widehat{G}$ generated by $\sigma(f)$. Of course, $\Gamma$ is countable and torsion-free. Hence there exists a monomorphism $h$ from $\Gamma$ into the group of reals (cf. [9, Theorem 8.1.2]). Changing, if necessary, the enumeration of the elements of $\sigma(f)$, we may assume that $h\left(\chi_{i}\right)<h\left(\chi_{j}\right)$ whenever $1 \leq i<j \leq n$. Since

$$
h\left(\chi_{n} \bar{\chi}_{1}\right)=h\left(\chi_{n}\right)-h\left(\chi_{1}\right)>h\left(\chi_{i}\right)-h\left(\chi_{j}\right)=h\left(\chi_{i} \bar{\chi}_{j}\right)
$$


whenever $(i, j) \neq(n, 1)(1 \leq i \leq n, 1 \leq j \leq n)$, it follows that the Fourier coefficient of $\sum_{i, j=1}^{n} a_{i} \bar{a}_{j} \chi_{i} \bar{\chi}_{j}$ at $\chi_{n} \bar{\chi}_{1}$ is equal to $a_{n} \bar{a}_{1}$. Moreover, since

$$
h\left(\chi_{n} \bar{\chi}_{1}\right)>h\left(\chi_{n} \bar{\chi}_{n}\right)=0,
$$

we see that $\chi_{n} \bar{\chi}_{1}$ is a non-trivial character of $G$. But

$$
\sum_{i, j=1}^{n} a_{i} \bar{a}_{j} \chi_{i} \bar{\chi}_{j}=\left|\sum_{i=1}^{n} a_{i} \chi_{i}\right|^{2}=1,
$$

so uniqueness of the Fourier expansion implies that $a_{n} \bar{a}_{1}=0$. This contradiction shows that $\sigma(f)$ is a singleton.

The proof is complete.

Passing to the characterization of $U_{0}(G)$ in the general case, we first show that the problem reduces to characterizing $U_{0}(G)$ for a compact Abelian group $G$ such that the component of 0 in $G$ (which is a closed subgroup of $G$ ) has finite index.

With $G$ an arbitrary locally compact Abelian group, let $f$ be an element of $U_{0}(G)$. Denote by $(\widehat{G})_{d}$ the group $\widehat{G}$ furnished with the discrete topology. Let $\Gamma$ be the subgroup of $(\widehat{G})_{d}$ generated by $\sigma(f), \operatorname{Per}(\Gamma)$ be the subgroup of $\Gamma$ consisting of all elements of finite order, and $H$ be the component of 0 in $\widehat{\Gamma}$. Then the dual of $\widehat{\Gamma} / H$ coincides with $\operatorname{Per}(\Gamma)$ (cf. [5, Corollary 24.20]). Since $\Gamma$ is finitely generated, it follows that $\operatorname{Per}(\Gamma)$ is finite and hence $H$ has finite index. Let $\alpha$ be the canonical homomorphism from $\Gamma$ into $\widehat{G}$. Then the dual homomorphism $\hat{\alpha}$, defined by

$$
(\hat{\alpha}(g), \chi)=(g, \alpha(\chi)) \quad(g \in G, \chi \in \Gamma),
$$

maps $G$ onto a dense subgroup of $\widehat{\Gamma}$. Moreover, there exists a unique $p$ in $U_{0}(\widehat{\Gamma})$ such that $f=p \circ \hat{\alpha}$. Thus it is clear that the passage from $f$ to $p$ yields the desired reduction.

Now we may and do assume that $G$ is a compact Abelian group such that the component $H$ of 0 in $G$ has finite index. Let $\left\{a_{i}: 1 \leq i \leq n\right\}$ be a subset of $G$ such that the sets $a_{i}+H \quad(1 \leq i \leq n)$ form the collection of all cosets of $H$ in $G$. We claim that

$$
\begin{aligned}
& U_{0}(G)=\left\{f \in \mathbb{T}^{G}: f\left(a_{i}+g\right)=c_{i} \chi_{i}(g) \text { for } g \in H,\right. \\
& \left.\qquad c_{i} \in \mathbb{T}, \chi_{i} \in \widehat{H}(1 \leq i \leq n)\right\},
\end{aligned}
$$

where $\mathbb{T}$ denotes the circle group.

Indeed, if we let $A$ denote the right-hand side set, the containment of $U_{0}(G)$ in $A$ follows from Proposition 3.1 and the fact that the 
dual of a connected locally compact Abelian group is torsion-free (cf. [5, Corollary 24.19]). Conversely, if $f \in A$, then

$$
\operatorname{span}\left\{T_{a} f: a \in G\right\} \subset \operatorname{span}\left\{\chi_{i} 1_{a_{j}+H}: 1 \leq i \leq n, 1 \leq j \leq n\right\},
$$

so $\operatorname{span}\left\{T_{a}: a \in G\right\}$ is finite dimensional, and hence $f$ is a trigonometric polynomial on $G$ (cf. [9, Theorem 7.8.3]). Thus $A \subset U_{0}(G)$ and the claim follows.

4. The main results. The starting point of our main considerations is the following.

THEOREM 4.1. Let $G$ be a compact Abelian group. Then

$$
U_{n}(G)=U_{0}(G)
$$

for each $n \in \mathbb{N}$.

Proof. Clearly, it suffices to prove that $U_{n}(G) \subset U_{0}(G)$ for each $n \in \mathbb{N}$. A simple induction argument shows that in fact it suffices to establish the containment of $U_{1}(G)$ in $U_{0}(G)$.

Given $f \in U_{1}(G)$, let $\Sigma$ be the subgroup of $\widehat{G}$ generated by $\sigma(f)$. Clearly, $\Sigma$ is countable. Let $\left(\sigma_{n}\right)_{n \in \mathbb{N}}$ be a family of finite subsets of $\Sigma$ such that $\sigma_{n} \subset \sigma_{n+1}$ for each $n \in \mathbb{N}$, and

$$
\Sigma=\bigcup_{n=1}^{\infty} \sigma_{n} .
$$

Given $n \in \mathbb{N}$, let

$$
F_{n}=\left\{a \in G: \sigma\left(\delta_{a} f\right) \subset \sigma_{n}\right\} .
$$

Each $F_{n}$ is clearly closed. Since, for each $a \in G, \sigma\left(\delta_{a} f\right)$ is a finite subset of $\Sigma$, it follows that

$$
G=\bigcup_{n=1}^{\infty} F_{n} .
$$

By Baire's theorem, there exist an open subset $V$ of $G$ and a positive integer $m$ such that $V \subset F_{m}$. By the compactness of $G$, there exists a finite subset $\left\{a_{i}: 1 \leq i \leq k\right\}$ of $G$ such that

$$
G=\bigcup_{i=1}^{\infty}\left(a_{i}+V\right) \text {. }
$$

For each $a \in G$, if $1 \leq i \leq k$ and $v \in V$ are such that $a=a_{i}+v$, then

$$
T_{a} f=T_{a_{\imath}}\left(\delta_{v} f\right) \cdot T_{a_{\imath}} f
$$


Thus

$$
\operatorname{span}\left\{T_{a} f: a \in G\right\} \subset \operatorname{span}\left\{\chi T_{a} f: \chi \in \sigma_{m}, 1 \leq i \leq k\right\} .
$$

Consequently, $\operatorname{span}\left\{T_{a} f: a \in G\right\}$ is finite dimensional, and hence $f$ is in $U_{0}(G)$.

The proof is complete.

LEMMA 4.2. Let $G$ be a compact Abelian group. Let $f$ be an almost unitary function in $G, S$ be a dense subset of $G$, and $n$ and $m$ be positive integers such that $\# \sigma\left(\delta_{s_{1} \cdots s_{n}} f\right) \leq m$ for any $s_{1}, \ldots, s_{n} \in S$. Then $f \in U_{0}(G)$.

Proof. Suppose that for some $a_{1}, \ldots, a_{n} \in G$, the spectrum of $\delta_{a_{1} \cdots a_{n}} f$ contains $m+1$ distinct elements $\chi_{1}, \ldots, \chi_{m+1}$. Then, in view of the continuity of the functions

$$
G^{n} \ni\left(b_{1}, \ldots, b_{n}\right) \rightarrow \mathscr{F} \delta_{b_{1} \cdots b_{n}} f\left(\chi_{i}\right) \quad(1 \leq i \leq m+1)
$$

and the denseness of $S$ in $G$, there exist $s_{1}, \ldots, s_{n} \in S$ such that

$$
\left\{\chi_{1}, \ldots, \chi_{m+1}\right\} \subset \sigma\left(\delta_{a_{1} \cdots s_{n}} f\right),
$$

a contradiction. Thus $f \in U_{m, n}(G)$, and hence, by the preceding theorem, $f \in U_{0}(G)$.

The proof is complete.

The next theorem is the main result of this section.

THEOREM 4.3. Let $G$ be a locally compact Abelian group. Then

$$
U_{n, m}(G) \subset U_{0}(G) \cup T E_{0}(G)
$$

for each $n \in \mathbb{N} \cup\{0\}$ and each $m \in \mathbb{N}$.

Proof. We shall proceed by induction on $n$ with $m$ arbitrarily fixed. The case $n=0$ is obvious.

Assume the assertion for $n-1$. Suppose that $f \in U_{n, m}(G) \backslash T E_{0}(G)$. Then there exist $\chi \in \widehat{G}$ and a Banach mean $m$ on $L^{\infty}(G)$ such that $\mathscr{F}_{m} f(\chi) \neq 0$. Let $h=f \bar{\chi}$. Then, clearly, $m(h) \neq 0$. Moreover, for each $a \in G, \delta_{a} h \in U_{n-1, m}(G)$, and hence, by the inductive hypothesis, either $\delta_{a} h \in U_{0}(G)$ or $\delta_{a} h \in T E_{0}(G)$. Since, for each $a \in G$,

$$
\delta_{-a} h=T_{-a} \delta_{a} \bar{h}
$$

and, for any $a, b \in G$,

$$
\delta_{a+b} h=\delta_{a} h \cdot T_{a} \delta_{b} h
$$


it follows that

$$
G_{0}=\left\{a \in G: \delta_{a} h \in U_{0}(G)\right\}
$$

is a subgroup of $G$. We claim that the index of $G_{0}$ is finite.

Suppose, on the contrary, that there exists an infinite subset $\left\{a_{n}: n \in\right.$ $\mathbb{N}\}$ of $G$ such that $a_{n}-a_{m} \notin G_{0}$ whenever $n \neq m$. Then, if $n \neq m$, then $\delta_{a_{n}-a_{m}} h$ is in $T E_{0}(G)$, and hence

$$
m\left(\delta_{a_{n}} \bar{h} \cdot \delta_{a_{m}} h\right)=m\left(T_{a_{m}} \delta_{a_{n}-a_{m}} \bar{h}\right)=m\left(\delta_{a_{n}-a_{m}} \bar{h}\right)=0 .
$$

We see that the image of $\left\{\delta_{a_{n}} \bar{h}: n \in \mathbb{N}\right\}$ by the canonical mapping from $L^{\infty}(G)$ onto the pre-Hilbert space

$$
H_{m}(G)=L^{\infty}(G) /\left\{f \in L^{\infty}(G): m\left(|f|^{2}\right)=0\right\}
$$

is an orthonormal set. For each $n \in \mathbb{N}$, we have

$$
m(h)=m\left(T_{a_{n}} h\right)=m\left(h \cdot \delta_{a_{n}} h\right) .
$$

Thus the Fourier coefficients of the image of $h$ in $H_{m}(G)$ relative to the image of $\left\{\delta_{a_{n}} \bar{h}: n \in \mathbb{N}\right\}$ in $H_{m}(G)$ are equal to $m(h)$, and hence, by Bessel's inequality, $m(h)=0$. This contradiction establishes the claim.

Let $b G$ be the Bohr compactification of $G$ and $\alpha: G \rightarrow b G$ be the canonical monomorphism from $G$ into $b G$. For each $\chi \in \widehat{G}$, let $\tilde{\chi}$ be the continuous character of $b G$ such that $\tilde{\chi} \circ \alpha=\chi$. As is known, the Fourier transformation sets up a one-to-one correspondence between $L^{2}(b G)$ and $l^{2}(\widehat{G})\left(=L^{2}\left((\widehat{G})_{d}\right)\right)$. Since by Bessel's inequality, the function

$$
\widehat{G} \ni \chi \rightarrow \mathscr{F}_{m} f(\chi) \in \mathbb{C}
$$

is in $l^{2}(\widehat{G})$, there exists a unique element $X$ in $L^{2}(b G)$ such that

$$
\mathscr{F} X(\tilde{\chi})=\mathscr{F}_{m} f(\chi) \quad(\chi \in \widehat{G}) \text {. }
$$

Since

$$
G_{0}=\left\{a \in G: \delta_{a} f \in U_{0}(G)\right\},
$$

it follows that for each $a \in G_{0}$, there exists a unique unitary trigonometric polynomial $P_{a}$ on $b G$ such that

$$
\delta_{a} f=P_{a} \circ \alpha \quad \lambda_{G} \text {-a.e. }
$$

If, for each $a \in G_{0}$, we let

$$
P_{a}=\sum_{\gamma \in \hat{G}} b_{\alpha, \gamma} \tilde{\gamma}
$$


then, in view of (4.1), for each $\chi \in \widehat{G}$,

$$
\begin{aligned}
\mathscr{F} T_{\alpha(a)} X(\tilde{\chi}) & =(\alpha(a), \tilde{\chi}) \mathscr{F} X(\tilde{\chi})=(a, \chi) \mathscr{F}_{m} f(\chi) \\
& =\mathscr{F}_{m} T_{a} f(\chi)=\mathscr{F}_{m}\left(f \delta_{a} f\right)(\chi) \\
& =\sum_{\gamma \in \hat{G}} b_{a, \gamma} \mathscr{F}_{m} f(\tilde{\chi}-\tilde{\gamma}) \\
& =\sum_{\gamma \in \hat{G}} b_{a, \gamma} \mathscr{F} X(\tilde{\chi}-\tilde{\gamma})=\mathscr{F}\left(X P_{a}\right)(\tilde{\chi})
\end{aligned}
$$

whence

$$
T_{\alpha(a)} X=X P_{a} \quad \lambda_{b G} \text {-a.e. }
$$

Let $\left\{a_{i}: 1 \leq k\right\}$ be a subset of $G$ such that the sets $a_{i}+G_{0} \quad(1 \leq$ $i \leq k)$ form the collection of all cosets of $G_{0}$ in $G$. Since

$$
b G \supset \bigcup_{i=1}^{k}\left(\alpha\left(a_{i}\right)+\overline{\alpha\left(G_{0}\right)}\right) \supset \overline{\bigcup_{i=1}^{k} \alpha\left(a_{i}+G_{0}\right)}=b G,
$$

the closures being taken in $b G$, it follows that the index of $\overline{\alpha\left(G_{0}\right)}$ in $b G$ is no greater than $k$. Thus $\overline{\alpha\left(G_{0}\right)}$ is an open subgroup of $b G$ and, in particular, the Haar measure in $\overline{\alpha\left(G_{0}\right)}$ is, to within normalization, the restriction to $\overline{\alpha\left(G_{0}\right)}$ of the Haar measure in $b G$.

Let $\left\{b_{j}: 1 \leq j \leq l\right\}$ be a subset of $\left\{a_{i}: 1 \leq i \leq k\right\}$ such that the sets $\alpha\left(b_{j}\right)+\overline{\alpha\left(G_{0}\right)}(1 \leq j \leq l)$ form the collection of all cosets of $\overline{\alpha\left(G_{0}\right)}$ in $b G$. For each $1 \leq j \leq l$, let $X_{j}$ denote the restriction of $T_{\alpha(b,)} X$ to $\overline{\alpha\left(G_{0}\right)}$. In view of $(4.3)$, for each $1 \leq j \leq l$ and each $a \in G_{0}$,

$$
T_{\alpha(a)}\left|X_{j}\right|=\left|X_{j}\right| \quad \lambda \overline{\alpha\left(G_{0}\right)} \text {-a.e. }
$$

Applying the Fourier transformation to both sides of the latter equality, we readily find that for $1 \leq j \leq l,\left|X_{j}\right|$ is $\lambda \overline{\alpha\left(G_{0}\right)}$-essentially constant. Choose $j_{0}$ so that

$$
X_{j_{0}} \neq 0 \quad \lambda \overline{\alpha\left(G_{0}\right)} \text {-a.e. }
$$

and set

$$
Y=\left|X_{j_{0}}\right|^{-1} X_{j_{0}}
$$

For each $a \in G_{0}$, let $R_{a}$ denote the restriction of $T_{\alpha\left(b_{J_{0}}\right)} P_{a}$ to $\overline{\alpha\left(G_{0}\right)}$. Since, by (4.3), for each $a \in G_{0}$,

$$
\delta_{\alpha(a)} Y=R_{a} \quad \lambda_{\overline{\alpha\left(G_{0}\right)}} \text {-a.e., }
$$

it follows from Lemma 4.2 that $Y \in U_{0}\left(\overline{\alpha\left(G_{0}\right)}\right)$. Hence in particular 
the set

$$
\Gamma=\left\{\gamma \in\left(\overline{\alpha\left(G_{0}\right)}\right)^{\wedge}: \gamma=\gamma_{1} \bar{\gamma}_{2} \text { for } \gamma_{1}, \gamma_{2} \in \sigma(Y)\right\}
$$

is finite.

Let $\left(\alpha\left(G_{0}\right)\right)^{\perp}$ be the annihilator of $\alpha\left(G_{0}\right)$ in $(b G)^{\wedge}$, that is, the set

$$
\left\{\gamma \in(b G)^{\wedge}:(\alpha(a), \gamma)=1 \text { for } a \in G_{0}\right\} .
$$

Being the dual of the quotient group $b G / \overline{\alpha\left(G_{0}\right)}$, the group $\left(\alpha\left(G_{0}\right)\right)^{\perp}$ is finite. Let $\pi$ be the canonical homomorphism from $(b G)^{-}$onto $\left(\alpha\left(G_{0}\right)\right)^{\wedge}$. Since the kernel of $\pi$ coincides with $\left(\alpha\left(G_{0}\right)\right)^{\perp}$, we see that the set $\pi^{-1}(\Gamma)$ is finite, and hence the set

$$
\Xi=\left\{\chi \in \widehat{G}: \chi=\gamma \circ \alpha \text { for } \gamma \in \pi^{-1}(\Gamma)\right\}
$$

is also finite.

In view of (4.4), $\Gamma$ contains the spectra of all the $R_{a} \quad\left(a \in G_{0}\right)$. Consequently, $\pi^{-1}(\Gamma)$ contains the spectra of all the $T_{\alpha\left(b_{j_{0}}\right)} P_{a} \quad(a \in$ $\left.G_{0}\right)$, and hence the spectra of all the $P_{a}\left(a \in G_{0}\right)$. Now Eq. (4.2) implies that

$$
\left\{T_{a} f: a \in G_{0}\right\} \subset \operatorname{span}\{\chi f: \chi \in \Xi\}
$$

whence

$$
\left\{T_{a} f: a \in G\right\} \subset \operatorname{span}\left\{\chi T_{a} f: \chi \in \Xi, 1 \leq i \leq k\right\} .
$$

We see that $\operatorname{span}\left\{T_{a} f: a \in G\right\}$ is finite dimensional, and so $f$ is in $U_{0}(G)$.

The proof is complete.

5. Applications. Let $R$ be a locally compact commutative ring, $\mathscr{R}$ be the additive group of $R, \chi$ be an element of $\widehat{\mathscr{R}}$, and $p$ be the function from $\mathscr{R}^{n}(n \in \mathbb{N})$ into $\mathscr{R}$ induced by a polynomial

$$
\sum_{|\alpha| \leq k} a_{\alpha} x^{\alpha} \quad\left(\alpha=\left(\alpha_{1}, \ldots, \alpha_{n}\right) \in(\mathbb{N} \cup\{0\})^{n},|\alpha|=\alpha_{1}+\cdots+\alpha_{n}\right)
$$

of $n$ variables with coefficients in $R$, of degree $k$. Then, of course, $\chi \circ p$ is in $U_{k, 1}\left(\mathscr{R}^{n}\right)$. Applying Theorem 4.3 to $\chi \circ p$, we obtain the following.

THEOREM 5.1. The function $\chi \circ p$ is an element either of $U_{0}\left(\mathscr{R}^{n}\right)$ or of $T E_{0}\left(\mathscr{R}^{n}\right)$.

Let

$$
R^{(2)}=\{r \in R: r=s t \text { for } s, t \in \mathbb{R}\} \text {. }
$$


THEOREM 5.2. Suppose that $R=R^{(2)}$, that $\widehat{\mathscr{R}}$ is torsion-free, that $k$ is not less than 2 , and that for some $\alpha$ with $|\alpha|=k$, the character $r \rightarrow \chi\left(a_{\alpha} r\right)$ of $\mathscr{R}$ is non-trivial. Then $\chi \circ p$ is in $T E_{0}\left(\mathscr{R}^{n}\right)$.

Proof. Suppose, on the contrary, that $\chi \circ p$ is not in $T E_{0}\left(\mathscr{R}^{n}\right)$. Let $\alpha=\left(\alpha_{1}, \ldots, \alpha_{n}\right)$ be a multi-index with $|\alpha|=k$ such that the character $r \rightarrow \chi\left(a_{\alpha} r\right)$ of $\mathscr{R}$ is non-trivial. Given $r_{1}, \ldots, r_{k} \in R$, put

$$
\begin{aligned}
a_{1} & =\left(r_{1}, 0, \ldots, 0\right), \\
a_{\alpha_{1}} & =\left(r_{\alpha_{1}}, 0, \ldots, 0\right), \\
a_{\alpha_{1}+1} & =\left(0, r_{\alpha_{1}+1}, \ldots, 0\right), \\
a_{\alpha_{1}+\alpha_{2}} & =\left(0, r_{\alpha_{1}+\alpha_{2}}, \ldots, 0\right), \\
a_{k} & =\left(0, \ldots, 0, r_{k}\right) .
\end{aligned}
$$

A straightforward calculation shows that

$$
\delta_{a_{1} \cdots a_{k}}(\chi \circ p)=\chi\left(\alpha ! a_{\alpha} r_{1} \cdots r_{k}\right) \quad\left(\alpha !=\alpha_{r} ! \cdots \alpha_{n} !\right) .
$$

Now Proposition 3.1, Theorem 4.3, and the fact that $\widehat{\mathscr{R}}$ is torsionfree imply that $\chi \circ p$ is in $U_{0,1}\left(\mathscr{R}^{n}\right)$. Since $k \geq 2$, it follows that $\delta_{a_{1} \cdots a_{k}}(\chi \circ p)=1$ and, consequently, that $\chi\left(\alpha ! a_{\alpha} r_{1} \cdots r_{k}\right)=1$ for any $r_{1}, \ldots, r_{k} \in R$. Taking into account that $R=R^{(2)}$ and that $\widehat{\mathscr{R}}$ is torsion-free, we infer that $\chi\left(a_{\alpha} r\right)=1$ for each $r \in R$, a contradiction.

The proof is complete.

As an immediate consequence of Theorem 5.2, we get the following generalization of a result of [1]:

THEOREM 5.3. Let $K$ be a locally compact commutative field, $\mathscr{K}$ be the additive group of $K, \chi$ be an element of $\widehat{\mathscr{K}}$, and $p$ be the function from $\mathscr{K}^{n}(n \in \mathbb{N})$ into $\mathscr{K}$ induced by a polynomial of $n$ variables with coefficients in $K$, of degree not less than 2. Then $\chi \circ p$ is in $T E_{0}\left(\mathscr{K}^{n}\right)$.

6. A counter-example. In this section we show that Theorem 4.3 fails in general if in the statement the set $U_{n, m}(G)$ is replaced by the set $U_{n}(G)$.

For each $n \in \mathbb{N}$, let $G_{n}$ be a non-zero finite Abelian group with a pair number of elements. Let $G$ be the direct sum of the $G_{n}(n \in \mathbb{N})$, and $\Sigma$ be the direct product of the $G_{n}(n \in \mathbb{N})$. Endow $G$ with the discrete topology, and $\Sigma$ with the product topology (of course, each $G_{n}$ is given the discrete topology). For each $n \in \mathbb{N}$, let $\pi_{n}$ be the canonical projection from $G$ onto $G_{n}$, and $\rho_{n}$ be the canonical 
projection from $\Sigma$ onto $G_{n}$. Let $\alpha$ be the canonical monomorphism from $G$ into $\Sigma$. Given $n \in \mathbb{N}$, let $e_{n}$ be a function from $G_{n}$ onto $\{-1,1\}$ such that

$$
\sum_{g \in G_{n}} e_{n}(g)=0
$$

and put

$$
f_{n}=e_{n} \circ \rho_{n}
$$

Let $\left(a_{n}\right)_{n \in \mathbb{N}}$ be a sequence of real numbers such that

$$
\sum_{n=1}^{\infty}\left|a_{n}\right|^{2}<+\infty, \quad \sum_{n=1}^{\infty}\left|a_{n}\right|=+\infty,
$$

and $\left|a_{n}\right|<\pi / 4$ for each $n \in \mathbb{N}$. Given $\sigma \in \Sigma$ and $a \in G$, set

$$
A(\sigma, a)=\exp \left[i \sum_{n=1}^{\infty} a_{n}\left(f_{n}(\sigma)-f_{n}(\sigma+\alpha(a))\right)\right] \text {. }
$$

To see that the above definition makes sense, note that given $a \in G$, there exists $m \in \mathbb{N}$ such that $\pi_{n}(a)=0$ whenever $n>m$, and so, for each $\sigma \in \Sigma$,

$$
A(\sigma, a)=\exp \left[i \sum_{n=1}^{m}\left(f_{n}(\sigma)-f_{n}(\sigma+\alpha(a))\right)\right] .
$$

One verifies at once that the mapping $A:(\sigma, a) \rightarrow A(\sigma, a)$ is a Borel unitary function on $\Sigma \times G$ satisfying

$$
A(\sigma, a+b)=A(\sigma, a) A(\sigma+\alpha(a), b)
$$

for all $\sigma \in \Sigma$ and all $a, b \in G . A$ is an example of what is called a cocycle on $\Sigma$ (cf. $[2,3,4]$ ).

Since, clearly, $\left(f_{n}\right)_{n \in \mathbb{N}}$ is a Bernoulli sequence on the probability triple $\left(\Sigma, \mathscr{B}(\Sigma), \lambda_{\Sigma}\right)$, where $\mathscr{B}(\Sigma)$ stands for the Borel $\sigma$-algebra of $\Sigma$ and $\lambda_{\Sigma}$ is the normalized Haar measure in $\Sigma$, it follows that the series $\sum_{n=1}^{\infty} a_{n} f_{n}(\sigma)$ converges for $\lambda_{\Sigma}$-almost all $\sigma$ in $\Sigma$. Let $Z$ be a real Borel function on $\Sigma \lambda_{\Sigma}$-almost everywhere equal to the sum of the above series. On putting

$$
Y=\exp (i Z),
$$

we see that given $a \in G$, the identity

$$
A(\sigma, a)=Y(\sigma) \overline{Y(\sigma+\alpha(a))}
$$

holds for $\lambda_{\Sigma}$-almost all $\sigma$ in $\Sigma$. The existence of a representation of $A$ as above is usually expressed as saying that $A$ is a coboundary. 
Each function of the form $a \rightarrow A(\sigma, a)(\sigma \in \Sigma)$ is called a trajectory of $A$. In view of (5.1), for each $a \in G$, the function $\sigma \rightarrow A(\sigma, a)$ is a unitary trigonometric polynomial on $\Sigma$. Hence, for each $\sigma \in \Sigma$ and each $b \in G$, the function $a \rightarrow A(\sigma+\alpha(a), b)$ is a unitary trigonometric polynomial on $G$. Taking into account the identity

$$
\overline{A(\sigma, a)} A(\sigma, a+b)=A(\sigma+\alpha(a), b) \quad(\sigma \in \Sigma, \quad a, b \in G),
$$

we thus see that each trajectory of $A$ is in $U_{1}(G)$. On the other hand, a modification of an argument used in the proof to [2, Theorem 2.4] shows that if some trajectory of $A$ is totally ergodic, then $A$ is a so-called $c$-coboundary, that is, there exists a unitary continuous function $X$ on $\Sigma$ such that

$$
A(\sigma, a)=X(\sigma) \overline{X(\sigma+\alpha(a))}
$$

for each $\sigma \in \Sigma$ and each $a \in G$. Below we shall show that $A$ is not a $c$-coboundary. Consequently, each trajectory of $A$ will provide an example of an element of $U_{1}(G)$ that is not in $U_{0}(G) \cup T E_{0}(G)$.

To show that $A$ is not a $c$-coboundary, suppose, contrariwise, that there exists a unitary continuous function $X$ on $\Sigma$ satisfying (6.4). Then in view of (6.3), given $a \in G$, the identity

$$
Y(\sigma+\alpha(a) \overline{X(\sigma+\alpha(a))}=Y(\sigma) \overline{X(\sigma)}
$$

holds for $\lambda_{\Sigma}$-almost all $\sigma$ in $\Sigma$. Applying the Fourier transformation to both sides of the latter equality, we see that there exist $c \in \mathbb{T}$ such that

$$
Y(\sigma)=c X(\sigma)
$$

for $\lambda_{\Sigma}$-almost all $\sigma$ in $\Sigma$.

Let $M$ be a positive number such that

$$
|z| \leq M\left|e^{z}-1\right|
$$

for each complex number $z$ with $|z|<\pi$. Since $\Sigma$ is compact, it follows that $X$ is uniformly continuous, and so there exists $k \in \mathbb{N}$ such that if $\sigma$ is in

$$
U_{k}=\left\{\theta \in \Sigma: \pi_{n}(\theta)=0 \text { for } n<k\right\},
$$

then

$$
\left\|T_{\sigma} X-X\right\|_{\infty}<\pi / 2 M
$$


where $\|\cdot\|_{\infty}$ denotes the supremum norm. For each $n \geq k$, let $\mathscr{A}_{n}$ be the $\sigma$-subalgebra of $\mathscr{B}(\Sigma)$ generated by the $f_{j}$ with $k \leq j \leq n$. Then, by (6.7), for each $n \geq k$ and each $\sigma \in U_{k}$,

$$
\left\|\mathbb{E}^{\mathscr{A}_{n}} T_{\sigma} X-\mathbb{E}^{\mathscr{A}_{n}} X\right\|_{\infty}<\pi / 2 M
$$

whence, in view of (6.2), (6.3), and (6.5),

$$
\left\|\exp \left[i \sum_{j=k}^{m} a_{j}\left(T_{\sigma} f_{j}-f_{j}\right)\right]-1\right\|_{\infty}<\pi / 2 M
$$

Proceeding by induction on $n$, we show now that for each $n \geq k$ and each $\sigma \in U_{k}$,

$$
\left\|\sum_{j=k}^{n} a_{j}\left(T_{\sigma} f_{j}-f_{j}\right)\right\|_{\infty}<\pi / 2 .
$$

For $n=k$, the inequality follows from the estimates

$$
\left\|a_{k}\left(T_{\sigma} f_{k}-f_{k}\right)\right\|_{\infty} \leq 2\left|a_{k}\right|<\pi / 2 .
$$

Assume the validity of the inequality for $n-1 \geq k$. Then

$$
\left\|\sum_{j=k}^{n} a_{j}\left(T_{\sigma} f_{j}-f_{j}\right)\right\|_{\infty}<\pi / 2+2\left|a_{n}\right|<\pi
$$

and now (6.9) results from (6.6) and (6.8).

Choose $\theta$ in $\Sigma$ so that the series $\sum_{j=1}^{\infty} a_{j} f_{j}(\theta)$ converges. Then, in view of (6.9), for each $n \geq k$ and each $\sigma \in U_{k}$,

$$
\left|\sum_{j=k}^{n} a_{j} f_{j}(\sigma+\theta)\right|<\pi / 2+\sup \left\{\left|\sum_{j=k}^{m} a_{j} f_{j}(\theta)\right|: m \geq k\right\} .
$$

On the other hand, it is easily seen that for each $n \geq k$,

$$
\sup \left\{\left|\sum_{j=k}^{n} a_{j} f_{j}(\sigma+\theta)\right|: \sigma \in U_{k}\right\}=\sum_{j=k}^{n}\left|a_{j}\right| .
$$

The last two relations show that $\left(a_{n}\right)_{n \in \mathbb{N}}$ is summable, a contradiction. Thus $A$ is not a $c$-coboundary, as was to be shown.

Acknowledgment. The author expresses his sincere thanks to Professor John Boris Miller for inviting him to stay at Monash University, 
Clayton, Victoria, Australia during the first semester in 1989 and gratefully acknowledges the excellent hospitality offered by the Department of Mathematics.

\section{REFERENCES}

[1] W. Chojnacki, Sur certains éléments ergodiques dans $l^{\infty}(G)$, Colloq. Math., 50 (1985), 121-124.

[2] _ Cocycles and almost periodicity, J. London Math. Soc., (2) 35 (1987), 98108.

[3] _-, Some non-trivial cocycles, J. Funct. Anal., 77 (1988), 9-31.

[4] H. Helson, Analyticity on compact Abelian groups, in Algebras in Analysis (J. H. Williamson, Ed.), pp. 1-62, Academic Press, New York/London, 1975.

[5] E. Hewitt and K. A. Ross, Abstract Harmonic Analysis, Vol. I, Spinger-Verlag, Berlin, 1963.

[6] J.-P. Pier, Amenable Locally Compact Groups, John Wiley \& Sons, New York, 1984.

[7] Fr. Lust-Piquard, Éléments ergodiques et totalement ergodiques dans $L^{\infty}(\Gamma)$, Studia Math., 69 (1981), 191-225.

[8] M. Talagrand, Some functions with a unique invariant mean, Proc. Amer. Math. Soc., 82 (1981), 253-256.

[9] W. Rudin, Fourier Analysis on Groups, Interscience, New York, 1962.

Received July 8, 1988 and in revised form April 7, 1989.

UNIVERSITY OF WARSAW

Palac Kultury i Nauki, IX P.

00-901 Warszawa, Poland

AND

MONASH UNIVERSITY

Clayton, Victoria 3168, Australia 


\section{PACIFIC JOURNAL OF MATHEMATICS EDITORS}

\author{
V. S. VARADARAJAN \\ (Managing Editor) \\ University of California \\ Los Angeles, CA 90024-1555-05 \\ Herbert Clemens \\ University of Utah \\ Salt Lake City, UT 84112 \\ Thomas ENRIGHT \\ University of California, San Diego \\ La Jolla, CA 92093
}

R. FINN

Stanford University

Stanford, CA 94305

Hermann FlaschKa

University of Arizona

Tucson, AZ 85721

VAUGHAN F. R. Jones

University of California

Berkeley, CA 94720

STEVEN KERCKHOFF

Stanford University

Stanford, CA 94305
C. C. MOORE

University of California

Berkeley, CA 94720

Martin SCharlemanN

University of California

Santa Barbara, CA 93106

HAROLd STARK

University of California, San Diego

La Jolla, CA 92093

\section{ASSOCIATE EDITORS}
R. ARENS
E. F. BECKENBACH
B. H. NeumanN
F. WoLF
(1904-1989)
K. YoshidA (1906-1982)

\section{SUP}

UNIVERSITY OF ARIZONA

UNIVERSITY OF BRITISH COLUMBIA

CALIFORNIA INSTITUTE OF TECHNOLOGY

UNIVERSITY OF CALIFORNIA

MONTANA STATE UNIVERSITY

UNIVERSITY OF NEVADA, RENO

NEW MEXICO STATE UNIVERSITY

OREGON STATE UNIVERSITY
UNIVERSITY OF OREGON

UNIVERSITY OF SOUTHERN CALIFORNIA

STANFORD UNIVERSITY

UNIVERSITY OF HAWAII

UNIVERSITY OF TOKYO

UNIVERSITY OF UTAH

WASHINGTON STATE UNIVERSITY

UNIVERSITY OF WASHINGTON 


\section{Pacific Journal of Mathematics}

Vol. 144, No. $1 \quad$ May, 1990

Wojciech Chojnacki, On some totally ergodic functions $\ldots \ldots \ldots \ldots \ldots \ldots 1$

Steven R. Costenoble, Stefan Waner and G. S. Wells, Approximating

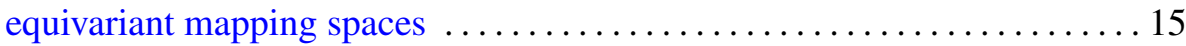

Peter Michael Higgins, A short proof of Isbell's zigzag theorem . . . . . . . 47

Harold H. Johnson, The absolute invariance of conservation laws ........5 51

Edgar Kann, Infinitesimal rigidity of almost-convex oriented polyhedra of arbitrary Euler characteristic $\ldots \ldots \ldots \ldots \ldots \ldots \ldots \ldots \ldots \ldots \ldots \ldots \ldots$

Alan Van Lair, Uniqueness for a nonlinear abstract Cauchy problem . . . . . 105

John B. Little and Kathryn A. Furio, On the distribution of Weierstrass points on irreducible rational nodal curves $\ldots \ldots \ldots \ldots \ldots \ldots \ldots \ldots \ldots 131$

J. S. Okon and Louis Jackson Ratliff, Jr., Reductions of filtrations . . . . . 137

Janusz Pawlikowski, Small subset of the plane which almost contains almost all Borel functions ............................... 155

Sergio A. Tozoni, Vector singular integral operators on a local field ....... 161 John Bason Wagoner, Triangle identities and symmetries of a subshift of

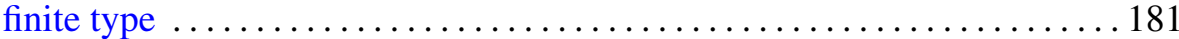

